\title{
DRE-Enhanced Swept-Wing Natural Laminar Flow at High Reynolds Numbers
}

\author{
Mujeeb Malik ${ }^{*}$ \\ Computational AeroSciences Branch, NASA Langley Research Center, Hampton, VA 23681 \\ Wei Liao ${ }^{\dagger}$ \\ National Institute of Aerospace, Hampton, VA 23666 \\ Fei Li ${ }^{\ddagger}$, Meelan Choudhari ${ }^{\S}$ \\ Computational AeroSciences Branch, NASA Langley Research Center, Hampton, VA 23681
}

Nonlinear parabolized stability equations and secondary instability analyses are used to provide a computational assessment of the potential use of the discrete roughness elements (DRE) technology for extending swept-wing natural laminar flow at chord Reynolds numbers relevant to transport aircraft. Computations performed for the boundary layer on a natural laminar flow airfoil with a leading-edge sweep angle of $34.6^{\circ}$, free-stream Mach number of 0.75 and chord Reynolds numbers of $17 \times 10^{6}, 24 \times 10^{6}$ and $30 \times 10^{6}$ suggest that DRE could delay laminar-turbulent transition by about $20 \%$ when transition is caused by stationary crossflow disturbances. Computations show that the introduction of small wavelength stationary crossflow disturbances (i.e., DRE) also suppresses the growth of most amplified traveling crossflow disturbances.

\section{Nomenclature}

$\begin{array}{lll}A_{i n i} & = & \text { initial disturbance amplitude } \\ C & = & \text { wing/airfoil chord length } \\ C_{L} & = & \text { lift coefficient } \\ C_{p} & = & \text { pressure coefficient } \\ f & = & \text { disturbance frequency } \\ H & = & \text { flight altitude } \\ M & = & \text { free stream Mach number } \\ m & = & \text { time Fourier mode number } \\ n & = & \text { spanwise Fourier mode number } \\ \mathrm{N} & = & \text { N-factor } \\ \mathrm{R}_{\mathrm{cf}} & = & \text { crossflow Reynolds number } \\ \mathrm{Re}_{\mathrm{c}} & = & \text { Reynolds number based on wing chord } \\ \mathrm{Tu} & = & \text { turbulence level in the free stream } \\ U_{c} & = & \text { velocity component in the crossflow direction } \\ U_{s} & = & \text { velocity in the inviscid streamline direction } \\ W_{m a x} & = & \text { maximum crossflow velocity in a crossflow velocity profile } \\ x & = & \text { normal-chordwise surface coordinate } \\ x_{0} & = & \text { normal-chord surface location where perturbation is initialized } \\ X & = & \text { normal-chordwise coordinate } \\ y & = & \text { wall normal coordinate } \\ Y & = & \text { distance from center of the aircraft fuselage } \\ Z & = & \text { spanwise coordinate in traveling frame of reference } \\ z & = & \text { spanwise coordinate } \\ \alpha_{m n} & = & \text { chordwise wavenumber of Fourier mode }(m, n) \\ \beta & = & \text { fundamental spanwise wavenumber }\end{array}$

\footnotetext{
* Senior Aerodynamicist, MS 128, Mujeeb.R.Malik@nasa.gov; Fellow, AIAA.

† Research Scientist, Wei.Liao@nasa.gov; Senior Member, AIAA.

\# Aerospace Technologist. MS 128, Fei.Li@nasa.gov.

$\S$ Aerospace Technologist, MS 128, Meelan.M.Choudhari@nasa.gov; Associate Fellow, AIAA.
} 


$\begin{array}{lll}\delta_{0.1} & = & \text { distance from wall where the crossflow velocity reduces to } 10 \% \text { of } W_{\max } \\ \phi & = & \text { any perturbation field variable } \\ \widehat{\phi} & = & \text { Fourier transform of } \phi \\ \xi & = & \text { dummy variable for normal-chordwise integration } \\ v_{e} & = & \text { kinematic viscosity at boundary-layer edge }\end{array}$

\section{Introduction}

Rin friction constitutes about 50\% of the drag budget of a business jet or a long haul transport aircraft (Ref. 1-3). Therefore, reduction in skin friction has the potential of yielding substantial savings in fuel burn. According to a system study (Ref. 3), a 10\% reduction in total skin friction drag would result in about $9 \%$ fuel savings for a 4000 $\mathrm{nm}$ transport aircraft. Since laminar skin friction is much less than the turbulent value, the subject of maintaining laminar flow for high Reynolds number flows (i.e., delay of boundary layer transition) has been of interest for well over half a century; see Ref. 4-8 for reviews of the subject.

In the low-amplitude free-stream disturbance environment typical of subsonic flight applications, transition in two-dimensional (2-D) boundary layers is caused by Tollmien-Schlichting (TS) instability which can be controlled by favorable pressure gradients, wall suction, and wall heat transfer (cooling in air, heating in water). Transition in three-dimensional (3-D) swept-wing boundary layers can also be caused by crossflow instability, which often manifests itself in the form of stationary co-rotating streamwise vortices that originate at minute roughness sites (Ref. 9). These vortices break down via a high frequency secondary instability mechanism (Ref. 10-14). Crossflow instability can be controlled by using wall suction, and experimental campaigns for demonstrating laminar flow control (LFC) using suction have been conducted in subsonic (Ref. 15-17) as well as in supersonic (Ref. 18) flight.

Crossflow instability results due to an inflection point in the crossflow velocity component (i.e., the component in the direction normal to the inviscid streamlines) and minimizing this velocity component would weaken the instability and yield extended runs of laminar flow. For a given leading-edge sweep angle and unit Reynolds number, the only way to reduce the crossflow velocity component is to minimize the chordwise pressure-gradient but that would bring the TS instability into play. Thus, swept-wing natural laminar flow (NLF) design is based upon striking a delicate balance by tailoring the pressure distribution such that the crossflow instability is reduced sufficiently while keeping TS growth at subcritical levels with respect to transition. One can only maintain this balance for a limited range of chord Reynolds numbers, so LFC via suction must be employed if laminar flow is desired at high Reynolds numbers associated with large transport aircraft.

The wavelength of crossflow disturbances scales with the boundary layer thickness, with small wavelength disturbances growing first (i.e., at small distance from the wing leading edge) but decaying over the mid-chord region. The longer wavelength disturbances become unstable later but remain unstable over longer streamwise distances and eventually break down via secondary instabilities that lead to laminar-turbulent transition. Saric et al.(Ref. 19) discovered that forcing small wavelength disturbances (i.e., the "control" mode) at relatively high amplitudes changes the boundary-layer mean flow such that the growth of more dominant larger wavelength disturbances (i.e., the "target" mode) is pushed downstream, thus delaying transition. This concept has been studied by using nonlinear parabolized stability equations (NPSE) (Ref. 20) and direct numerical simulations (DNS) (Ref. 21). Both these analyses support the soundness of the fundamental concept. In practice, the control mode is introduced via discrete spanwise periodic roughness elements placed near the neutral point of the instability diagram. These control disturbances only introduce harmonics and no subharmonics, and therefore do not directly feed into the larger wavelength target modes. This strategy for controlling crossflow disturbances is known as discrete roughness elements (DRE) technology and has the potential to increase the range of applicability of NLF to higher chord Reynolds numbers. Computations based on NPSE and secondary instability analysis have been performed for subsonic swept wing designs at Reynolds numbers of $7.15 \times 10^{6}$ and $17 \times 10^{6}$, which showed that DRE could delay the growth of the stationary target modes and the associated secondary instabilities at these Reynolds numbers (Ref. 22-23).

Saric et al. (Ref. 24-25) reported a flight experiment using Cessna O-2 aircraft to demonstrate the DRE concept. The test article consisted of a $30^{\circ}$ swept blade that was mounted vertically under one of the wings of the aircraft. The maximum transition Reynolds number obtained on the test article was about 6.4 million when the leading edge was 
polished with a surface finish quoted as $0.3 \mu \mathrm{m}$ root mean square (rms) and $2.2 \mu \mathrm{m}$ average peak-to-peak. Here, the transition Reynolds number was limited by a pressure minimum (i.e., adverse pressure gradient) because of the relatively low chord Reynolds number of 8 million. In other words, an increased extent of the NLF could be had for a larger test article. When the leading edge was painted to yield a deteriorated surface finish (quoted as $1 \mu \mathrm{m} \mathrm{rms,}$ with $3.8 \mu \mathrm{m}$ peak-to-peak), transition Reynolds number dropped to about 2.4 million owing to the adverse effect of surface roughness on transition; with the application of DRE, transition Reynolds number increased to about 4.8 million for the chord Reynolds number of 8 million. This is the highest Reynolds number at which the DRE technology has been demonstrated to delay crossflow-induced transition. Clearly, natural laminar flow could be maintained at such Reynolds numbers without the use of DRE, and there is a need to demonstrate the technology at higher chord Reynolds numbers, Mach numbers, and lift-coefficients of relevance to transport aircraft.

NASA's Environmentally Responsible Aviation (ERA) Project sponsored the evaluation of DRE as a laminar flow control technology for potential application to transport aircraft. A collaborative effort between Dryden Flight Research Center, Langley Research Center, and Texas A\&M University (TAMU) was initiated to design a flight experiment using Dryden's Gulfstream-III (G-III, hereinafter G-3) aircraft. A wing glove was designed by TAMU, with the glove leading-edge sweep of $34.6^{\circ}$ with a maximum possible chord Reynolds number approaching $30 \times 10^{6}$. The details of the glove design and analyses using computational fluid dynamics (CFD) and stability analyses codes have been given in Ref. 26-30.

This paper provides an assessment of the DRE applicability to high Reynolds number flows using nonlinear parabolized stability equations (NPSE) and secondary instability analysis, extending the previous results obtained for a different airfoil design in Ref. 23. The particular conditions used for the assessment consist of a free-stream Mach number of 0.75 and Reynolds numbers of $17 \times 10^{6}, 24 \times 10^{6}$ and $30 \times 10^{6}$. A small wavelength sub-critical stationary crossflow disturbance (i.e., the 'control' mode) is introduced to study its effect on the nonlinear evolution of the longer wavelength, most amplified stationary crossflow disturbances (i.e., the 'target' mode). Secondary instability analyses of the target mode with and without control were performed to provide an assessment of the ability of DRE to delay laminar-turbulent transition at high Reynolds numbers. Initial amplitudes of the target and control modes were assumed, and the modal shapes were taken from the stability analysis. Thus, the impact of the actual surface roughness to initiate natural crossflow disturbances as well as the control mode induced by DRE was not simulated during the present study.

Traveling disturbances of the crossflow type are also amplified by the boundary layer, and, in fact, these disturbances are known to be more unstable than the stationary crossflow disturbances. Traveling disturbances are sensitive to free-stream turbulence level, which is relatively low at aircraft flight altitudes. Therefore, traveling disturbances are considered to be much less relevant as compared to stationary disturbances, which are directly induced by surface roughness. Here, the results of NPSE computations are presented, which show that the growth of traveling disturbances is also suppressed by introducing smaller wavelength stationary crossflow disturbances (i.e., DRE).

\section{Computational "Test Article"}

\section{A. Laminar Flow Glove Design}

The goal set by the Environmentally Responsible Aviation Project was to demonstrate the DRE technology for a leading-edge sweep in excess of $30^{\circ}$ and chord Reynolds numbers that are characteristics of mid-range transport aircraft, i.e., $\mathrm{Re}_{\mathrm{c}}$ up to $30 \times 10^{6}$. The design of a laminar flow glove for the Gulfstream-III aircraft was described in Ref. 28. The glove has a leading-edge sweep of about $34.6^{\circ}$ compared to $31.7^{\circ}$ for the original wing. Figure 1 shows the glove as installed on the G-3 aircraft wing. The glove width is $6 \mathrm{ft}$ and the mid-span chord length, including the glove, is $14.5 \mathrm{ft}$. The Mach number range of interest is 0.66 to 0.75 , with cruise section $C_{L}$ of approximately 0.5 . Chord Reynolds numbers in the range of $15 \times 10^{6}$ to $30 \times 10^{6}$ could be achieved within the designed flight envelope. One of the objectives of the experiment was to demonstrate that DRE could delay transition by $50 \%$ as compared to the NLF case.

The flow conditions for the present computations are: $M=0.75, H=38,840 \mathrm{ft}$, angle of attack $(\mathrm{AoA})=3.5^{\circ}$. The resulting glove mid-span chord Reynolds number is $24.2 \times 10^{6}$; however, for convenience, it is identified as $24 \times 10^{6}$ elsewhere in the paper. In Ref. 29, both structured-grid (OVERFLOW, Ref. 31) and unstructured-grid (FUN3D, Ref. 32) Navier-Stokes codes were used to compute the aircraft flow-field including the wing glove. Figure 2 shows the 
upper surface $C_{p}$ distribution in the glove region. The three vertical lines ( $Y=204 \mathrm{in}, 234 \mathrm{in}$, and $\left.264 \mathrm{in}\right)$ in the plot indicate the distance from the center of the aircraft fuselage. The three spanwise lines indicate relative distance $X / C$ from the leading-edge, the line denoted $X / C=0.6$ being slightly ahead of the shock. In these computations, turbulent flow was assumed everywhere except on the glove upstream of the shock. It should be noted that the isobars in the glove region have sweep angles smaller than the constant $X / C$ lines, particularly at larger distances from the leadingedge. This unsweeping of the isobars has an important effect on boundary-layer stability as discussed in Ref. 29 and summarized below.

\section{B. Boundary-Layer Mean Flow}

Figure 3 shows the $C p$ distribution along the $Y=234$ inch butt-line. The favorable pressure distribution on the upper surface assures that Tollmien-Schlichting instability is minimized and crossflow instability is present due to the glove sweep. The upper surface pressure distribution along with the airfoil cross section along $Y=234$ inch is used in the laminar boundary layer code (Ref. 33) under the infinite-swept wing assumption, and the resulting mean flow profiles will be used in the analyses presented in the next section. However, it is important to point out the differences between this mean flow computed under the infinite-swept wing assumption and the fully 3-D mean flow present in the mid-span region of the glove. The 3-D boundary layer flow on the glove was extracted from the FUN3D and OVERFLOW viscous computations. A quantity that can be used to gauge the strength of crossflow instability is the crossflow Reynolds number defined as,

$$
R_{c f}=\frac{W_{\max } \delta_{0.1}}{v_{e}}
$$

where $W_{\max }$ is the maximum crossflow velocity, $\delta_{0.1}$ is the distance from the wall where the crossflow velocity reduces to $10 \%$ of its maximum value, and $v_{e}$ is the kinematic viscosity at the boundary layer edge.

Figure 4(a) shows a comparison of $R_{c f}$ computed from the boundary layer code, under infinite-swept wing assumption and the two Navier-Stokes codes. For the latter computations, boundary layer profiles were extracted from the steady laminar Navier-Stokes solutions to obtain the maximum crossflow velocity and the crossflow length scale at each station. The values of crossflow Reynolds numbers computed from FUN3D and OVERFLOW solutions are quite close, except that the former gives a nonsmooth distribution as the boundary layer profiles are linearly interpolated from the tetrahedral grid. Figure 4(b) shows the plots of an inviscid streamline computed from the two Navier-Stokes codes and the two streamlines essentially coincide. It is clear that the full 3-D mean flows from FUN3D and OVERFLOW codes yield much lower values of $R_{c}$, particularly away from the leading edge, and this is because the unsweeping of the isobars helps reduce the crossflow component of the velocity as compared to the infinite-swept assumption. The much lower values of crossflow will then result in much reduced growth rates for the crossflow instability. This is shown in Fig. 5, where $N$ factors are plotted for stationary crossflow disturbances of fixed spanwise wavelengths. These $N$ factors are computed using quasi-parallel linear stability theory (LST), as implemented in the LASTRAC code (Ref. 34). The mean flows used in these analyses are computed using the boundary layer code and the FUN3D code. The boundary layer solution, under the infinite-swept assumption, yields a maximum $N$ factor of about 20, which reduces to about 12 for the mean flow using FUN3D. This reduction in $N$ factor is expected because of substantial reduction in the $R_{c f}$ shown in Fig. 4(a). A similar reduction in the $N$ factor was noted when mean flow from the structured-grid OVERFLOW code was used.

The above results show that the as-designed G-3 glove boundary layer is much more stable than the boundary layer under the infinite-swept assumption using the same streamwise pressure distribution. However, to simplify the computations of nonlinear disturbance evolution, the infinite-swept-wing boundary layer solution was used in the computational results presented below. Because of the substantially higher linear growth in the infinite swept case, transition assessment in this context presents a more stringent test for the effectiveness of DREs in comparison with the actual boundary layer flow over the glove. The reader should note that the chord Reynolds number for the freestream conditions given above is $24 \times 10^{6}$. In the next section, stability results for two additional Reynolds numbers, namely $17 \times 10^{6}$ and $30 \times 10^{6}$, are presented but the boundary layer mean flows for these cases were computed with the same $C_{p}$ shown in Fig. 3 by simply changing the free-stream Reynolds number. 


\section{A. Selection of Target and Control Modes \\ III. Nonlinear Computations and Secondary Instability Analysis}

Calculations were first performed to determine the wavelength of the most amplified stationary (frequency $=0$ $\mathrm{Hz}$ ) crossflow disturbances, whose growth needs to be suppressed in order to delay transition. Since these are the targets of the DRE control, instability associated with this wavelength is designated as the target mode. Determination of the target mode wavelength is done by performing linear parabolized stability equations (LPSE) analysis, which includes surface curvature and nonparallel effects. Figure 6 presents the LPSE results for three Reynolds numbers $\left(17 \times 10^{6}, 24 \times 10^{6}\right.$ and $\left.30 \times 10^{6}\right)$. For demonstration purposes, the target mode wavelengths were selected to be $10 \mathrm{~mm}, 8 \mathrm{~mm}$, and $7 \mathrm{~mm}$ for the three Reynolds numbers, respectively. $N$ factors computed using LPSE for these wavelengths, and others, grow to large numbers between 11 and 20, approximately. The stationary crossflow disturbances associated with these wavelengths are the target modes for this control study and the subject of control using DRE. The results in Fig. 6 also show $N$ factors for smaller wavelength disturbances. For example, wavelengths of $2.67 \mathrm{~mm}$ and $4 \mathrm{~mm}$ reach maximum $N$ factors of 4 and 6.5, respectively, for $R e_{c}=24 \times 10^{6}$. A wavelength of $4 \mathrm{~mm}$ was selected as the control mode since the $2.67 \mathrm{~mm}$ mode was found to have a relatively weak effect on the target mode. The control modes with wavelengths of $5 \mathrm{~mm}$ and $2.33 \mathrm{~mm}$ are selected for the Reynolds numbers of $17 \times 10^{6}$ and $30 \times 10^{6}$, respectively.

\section{B. Nonlinear Evolution of Stationary Crossflow Disturbances with and without DRE control}

Nonlinear parabolized stability equations, as implemented in LASTRAC, were used to study the evolution of target modes with and without the control modes. For an infinite-swept wing, the NPSE solution for a general nonlinearly developing traveling crossflow wave with a fundamental frequency $f$ and fundamental spanwise wavenumber $\beta$ is given by

$$
\phi(x, y, z, t)=\sum_{m} \sum_{n} \hat{\phi}(x, y, m, n) \exp \left(i \int_{x_{0}}^{x} \alpha_{m n}(\xi) d \xi+i n \beta z-i m 2 \pi f t\right)
$$

where $\phi$ represents any perturbation field variable, $x, y$ and $\mathrm{z}$ are, respectively, the chordwise, wall-normal, and spanwise surface coordinates, and $\alpha_{m n}$ is the streamwise wavenumber of Fourier component $(m, n)$. Resolving a general nonlinear perturbation wave accurately requires many Fourier modes $(m, n)$, which makes the computation of traveling waves more time-consuming than that of stationary waves. However, to begin with, we present results for purely stationary modes, in which case $\mathrm{f}=m=0$ and Eq. 1 becomes:

$$
\phi(x, y, z)=\sum_{n} \widehat{\phi}(x, y, n) \exp \left(i \int_{x_{0}}^{x} \alpha_{0 n}(\xi) d \xi+i n \beta z\right)
$$

The initial mode shapes for the target and the control modes were determined from the LPSE analysis. These modes were assigned some arbitrary initial amplitude, which was very small for the target mode and relatively larger for the control mode. This is because the DRE control will induce relatively large amplitude of the smaller wavelength control mode as compared to the target mode, which is induced by the natural distributed surface roughness. Different initial amplitudes of the target modes are linked to the surface quality (e.g., polished vs. painted) and different initial amplitudes of the control mode to the height and diameter of the discrete roughness elements. Quantitative analysis for a specific experimental configuration would require the determination of boundary layer receptivity to the actual shape, height, and distribution of the roughness. Lacking a priori information concerning the roughness definition, the consideration of the receptivity phase is avoided in the current analysis, which makes the present results qualitative in nature. It is felt that DNS of the natural and imposed surface roughness will be required to draw definitive conclusions.

Throughout this paper the word "amplitude" will frequently be used, therefore, it is necessary to clarify its meaning here at the outset. Unless otherwise stated, the amplitude of the perturbation at a location on the wing is defined as the ratio of the local maximum of the chordwise perturbation velocity to the global freestream velocity. 
The results for the evolution of various modes in a nonlinear computation for $R e_{c}=24 \times 10^{6}$ are described first. To initiate the nonlinear computation, the mode shape is first computed for the target mode of wavelength $8 \mathrm{~mm}$ using LPSE. The chordwise velocity component for this mode is assigned an initial amplitude of $10^{-4}$, i.e., $.01 \%$ of the free-stream velocity. For stationary crossflow computations, the number of Fourier modes used in the spanwise direction is 40 , i.e. $-40<n<40$ in Eq. 2. Figure 7(a) presents the results for the case without control. The primary mode $(0,1)$ initially follows the linear PSE result until its harmonics grow to significant enough amplitudes for the primary mode to saturate, as indicated by the flattening out of the amplitudes in Fig. 7 (a). It can be seen that the disturbance energy cascades into the harmonics $(0,2),(0,3)$, etc. The mean flow distortion $(0,0)$ mode also gains significance, and it attains amplitude equivalent to the first harmonic (i.e., 0,2$)$ mode. These results are similar to the nonlinear crossflow disturbance evolution computed in Ref. 11 for a canonical problem and in Ref. 12 for a low speed swept-wing flow. The results for the case with DRE control using a control wavelength of $4 \mathrm{~mm}((0,2)$ mode $)$ are shown in Fig. 7(b), with an initial amplitude for the control mode of .015 (i.e., 1.5\% of the free-stream velocity). In contrast with the results in Fig. $7(a)$, initial amplitude of $(0,2)$ mode is much higher than the $(0,1)$ mode. It can be seen that the control mode initially grows but eventually decays within a short chordwise distance, as expected from the LPSE results. The target mode growth is delayed, as discussed in detail in the following paragraphs. Here, one should note that after a period of decay, the control mode picked up again further downstream as a harmonic of the target mode which, by then, had attained a large amplitude.

Figure 8 shows the results of a stationary crossflow vortex with and without control at $x / c=0.35$. Two spanwise periods are shown. The introduction of small wavelength control mode suppresses the development of the crossflow vortex, which can be clearly seen in Fig 8(b) when compared to Fig. 8(a), implying that transition induced by stationary crossflow mechanism will be delayed.

Results for crossflow disturbance evolution for three different Reynolds numbers $\left(17 \times 10^{6}, 24 \times 10^{6}\right.$ and $30 \times$ $10^{6}$ ), with and without DRE control, are shown in Figs. 9-11. In this case, only the amplitudes of target and control modes are shown. Target modes were assigned three different amplitudes of $10^{-5}, 10^{-4}$ and $10^{-3}$, qualitatively representing ultra-polished, polished, and painted surfaces, respectively. Initial amplitudes of the control modes were selected as 0 (i.e., no control), $.005, .01, .015$. For $R e_{c}=17 \times 10^{6}$, additional computations with a control amplitude of .02 were performed. Target mode growth was progressively delayed further downstream with increasing control amplitude in all cases.

In the results shown in Figs. 9-11, saturation amplitude of the target mode reached in excess of 0.5 (i.e, 50\%). In the low speed case analyzed in Ref. 12, the fundamental mode saturated at only about $20 \%$. In that case the amplitude was based on the ratio of the maximum perturbation local velocity to the boundary-layer edge velocity in the inviscid streamline direction. If the freestream velocity based amplitude of 50\% in the present analysis is converted to the boundary-layer edge velocity based amplitude, the value drops to approximately $40 \%$ mainly because the boundary layer-edge velocity is larger than the freestream velocity. This conversion enables comparisons to be made between the two cases. In the present high speed case, the saturation amplitude is approximately twice as large as it was in the low speed case. If one assumes that transition will occur when the disturbance amplitude exceeds 0.25 , then transition locations for the no control and control (amplitude $=.015$ ) cases can be estimated from these results and are given in Table 1 for the three Reynolds numbers and the three initial amplitudes of the target modes. It can be seen that, except for the lowest Reynolds number and the highest initial amplitude of target mode, DRE control (using initial amplitude $=0.015$ ) moves transition location $(X / C)$ by $20-25 \%$. The next section reports the estimated transition locations based on the secondary instability analysis. Here, one should note that transition delay using an initial amplitude of .015 for $R e_{c}=17 \times 10^{6}$ is smaller than $20 \%$. It requires initial amplitude of .02 to delay transition beyond $20 \%$ in this case (see Table 2). No attempt has been made to optimize the results with respect to the control mode wavelength, and the results in Tables 1 and 2 are likely to change if an optimization study were to be performed. However, it is felt that such a study would not add value without including the critical step of boundary layer receptivity to DRE and surface roughness.

\section{Secondary Instability of the Target and Control Modes}

As the stationary crossflow vortex amplitude increases, the nonlinear interactions among the many harmonics of the disturbance cause the crossflow vortex to reach large amplitude, quasi-saturated state, which leads to the appearance of strong shear-layers as can be inferred from results in Fig. 8(a). The crossflow vortex is now susceptible to secondary instability similar to that in the case of large amplitude Görtler vortices, Refs. 35 and 36. 
The secondary instability is similar to the primary instability in that it is also an unstable perturbation to some mean flow state. The main difference is that, for a primary instability wave, the mean flow varies strongly in the wallnormal direction only, but for a secondary instability wave, the mean flow also varies strongly in the spanwise direction; the streamwise variation in both cases is considered to be weak. The mathematical formulation of the former leads to an eigenvalue problem of a set of ordinary differential equations depending on a single spatial variable, while that of the latter gives rise to an eigenvalue problem of a set of partial differential equations depending on two spatial variables.

For the particular problem of secondary instability of stationary crossflow vortices, the mean flow varies strongly in the wall-normal direction and, owing to the infinite-swept assumption, is periodic in the spanwise direction. Along the crossflow vortex axis, the mean flow varies slowly, and, therefore, a parallel assumption can be made to ignore the mean flow changes so that an ansatz can be used to factor out the oscillatory component of the secondary instability wave in this direction. However, a problem unique to this flow configuration is that the direction of the crossflow vortex axis is not orthogonal to the spanwise direction. This somewhat complicates the mathematical formulation. To get around this problem, a non-orthogonal coordinate system was devised (see Refs. 37 and 38) that properly enforces the periodicity in the spanwise direction and the slow variation in the vortex axis direction, resulting in the correct normal-chordwise secondary instability growth rate.

The secondary instability code used in the current analyses has been validated against solutions of nonlinear PSE and direct numerical simulations based on a mean flow that is invariant along the vortex axis and a small initial amplitude perturbation at in-flow. The agreement was excellent.

In the numerical computations, fourth-order finite differences were used in the wall-normal direction, and the Fourier spectral method was used in the spanwise (periodic) direction with typically 121 and 32 points, respectively. Given that there are five equations in this problem, the total number of degrees of freedom of the resulting discretized system is $5 \times 121 \times 32=19360$. Grid convergence is confirmed by carrying out the eigenvalue computations using more grid points in each direction for selected points in the parameter space of the problem.

A typical secondary instability $N$ factor computation procedure can be summarized (see Refs. 37 and 38 for details) as follows:

1. Using the Arnoldi method, a thorough temporal eigenvalue search at a few selected streamwise stations was carried out to identify the relevant secondary instability modes and their frequency ranges.

2. A suitable representative subset of secondary instability modes was chosen to span the relevant frequency range. Each of these temporal eigenvalues was subject to iterations toward a spatial eigenvalue by fixing the real part of the eigenvalue (complex frequency) and changing the complex wavenumber until the imaginary part of the frequency became smaller than a preset tolerance. Inverse Rayleigh iteration method was employed in this step.

3. With each selected eigenvalue from the previous step as an initial guess, the eigenvalue computations were marched both upstream and downstream in the chordwise direction to cover the entire chordwise range of interest. Finally, $N$ factors are computed from the eigenvalues.

Secondary instability computations were performed for the three Reynolds numbers using the target mode amplitude of $10^{-4}$. Various control amplitudes between 0 and 0.015 were used. Figure 12 presents the secondary $N$ factor results for the target and control modes. Secondary instability $N$ factors for the target mode rise very sharply for all control amplitudes and Reynolds numbers. If secondary instability $N=9$ is selected to indicate the onset of transition, then the delay in boundary layer transition because of the DRE could be computed (Table 3). It can be seen that transition delay is between $20 \%$ and $23 \%$ for the three Reynolds numbers. Since the $N$ factor curves are essentially parallel, percent change in transition will remain the same regardless of the value of $N$ factor selected to indicate transition onset. It is also noted that transition delay predicted by secondary instability analysis is much more consistent for the three Reynolds numbers as compared to predictions based on fixed amplitude in Table 1 .

Secondary $N$ factors for the control modes are also presented in Fig. 12. For the 17 million Reynolds number case, the maximum $N$ factors are approximately the same (just under three) regardless of initial amplitudes. For the 
24 million Reynolds number case, the maximum $N$ factors are strongly dependent on initial amplitudes, and the largest of these reaches approximately 8.5. For the highest Reynolds number case of 30 million, the initial amplitude dependency of maximum $N$ factors is strong, but the maximum $N$ factor reached is only approximately three. This apparent lack of trend in the secondary instability $N$ factors for the control mode should not come as a surprise because the Reynolds number is not the only parameter that is different in these three cases. For the first two cases, the control mode wavelength is half that of the target mode, while for the last case, the control mode wavelength is only one-third that of the target mode. Different control mode wavelengths may give rise to different values of secondary $N$ factors and may also influence the nonlinear growth of the target modes as noted above.

One of the potential problems of the DRE control is that, if too much control is introduced, the control mode itself may lead to transition by attaining large enough amplitudes and, hence, becoming susceptible to secondary instability (Ref. 30). The secondary instability analysis results presented above in Fig. 12(b) for the $4 \mathrm{~mm}$ control mode with an initial amplitude of 0.015 (the largest used for the control mode) shows that the maximum $N$ factor of secondary instability reaches approximately 8.5 for a mode with a frequency of approximately $95 \mathrm{kHz}$. Secondary instability analysis using nonlinear PSE needs to be performed to determine whether this instability will lead to transition. In order to do this computation, the linear secondary instability mode at $X / C=0.12$ is Fourier decomposed in the z-direction into different $n \beta$ modes (see Eq. 1) and used as in-flow conditions (along with the stationary modes) for the nonlinear PSE analysis. Three different initial amplitudes for the secondary disturbances are used, namely, $10^{-6}, 10^{-5}$, and $10^{-4}$. The amplitude of the stationary control mode is fixed at .015 . As shown in Fig. 13, with the two smaller initial amplitudes, the secondary instability grows and dies without causing transition. With the third, higher initial amplitude, the secondary instability amplitudes exhibit some oscillatory behavior; however, an examination of the rms contours of the streamwise perturbation velocity shows no indication of the flow breaking down to smaller scales. Thus, transition due to the control mode is not expected in this case either.

The secondary instability results presented herein suggest that the DRE could delay stationary crossflow-induced transition by about $20 \%$ for all three Reynolds numbers as compared to the no-control swept-wing natural laminar flow case. Secondary instability analysis also yielded interesting results on the characteristics of secondary instability modes, but that discussion is deferred to a later section as their characteristics are not directly related to the main theme of the paper. The question of DRE based control in the presence of traveling crossflow disturbances is considered first.

\section{Effect of DRE on Traveling Crossflow Disturbances}

It is well known that traveling disturbances grow much more than the stationary ones and, if excited at a sufficiently high amplitude, would dominate laminar-turbulent transition in swept-wing flows. Based on the experimental data of Refs. 39 and 40, it was argued in Ref. 41 that transition is caused by stationary disturbances when $\mathrm{Tu} \sim .02 \%$ and dominated by traveling instability waves when $\mathrm{Tu} \sim .15 \%$. Since turbulence level at aircraft flight altitudes is low, in-flight swept-wing transition is expected to be caused by surface roughness-induced stationary crossflow disturbances, which can then be controlled using the DRE. By performing nonlinear PSE computations, it is shown here that the DRE could also delay the growth of traveling crossflow disturbances if present in the boundary layer.

As noted earlier, the NPSE solution for a general nonlinearly developing traveling crossflow wave of a fixed frequency $f$ and spanwise wavenumber $\beta$ is given by Eq. 1. Resolving a general nonlinear perturbation wave accurately requires many Fourier modes $(m, n)$, which makes the computation of traveling waves more timeconsuming than that of stationary waves. However, if a nonlinear perturbation develops from a single small amplitude wave initialized as Fourier mode $(1,1)$, the only harmonics that are nonlinearly generated as the wave amplitude grows are those on the diagonal of the $(m, n)$-plane, i.e., modes $(n, n)$, all other off-diagonal modes for which $m \neq n$ are 0 . Consequently, a coordinate transformation can be made as follows

$$
Z=z-(2 \pi f / \beta) t
$$

i.e., the new coordinate system travels spanwise with the spanwise phase speed of the perturbation. Upon substitution of Eq. 3 into Eq. 1, one has 


$$
\phi(x, y, Z)=\sum_{n} \widehat{\phi}(x, y, n) \exp \left(i \int_{x_{0}}^{x} \alpha_{0 n}(\xi) d \xi+i n \beta Z\right)
$$

Therefore, the problem now becomes one of stationary wave, resulting in an order of magnitude speed up in computational turnaround. For example, if a nonlinearly evolving traveling crossflow vortex requires 48 spatial Fourier modes to resolve in a traveling frame of reference, it will require $48 \times 48$ spatial and time Fourier modes to resolve the wave in the fixed frame of reference since these Fourier modes now lie along the diagonal of $(m, n)$ space, and Eq. 1 must be used to represent the perturbation. The coordinate transformation works best and results in the savings of memory and time when only a single traveling crossflow vortex is initialized; however, it also works in some specific cases in which both stationary and traveling crossflow vortices are present as exemplified in the following case.

If a stationary mode of shorter wavelength is introduced to control the traveling mode, then a transformation of the form given by Eq. 3 will not result in a stationary flow field. However, since the maximum amplitude of the control mode remains relatively small, fewer Fourier modes are required to accurately represent it. By applying the transformation in Eq. (3), the traveling target mode becomes stationary, and the stationary control mode becomes a traveling mode. A larger number of modes are used in $n$ to resolve the target mode, while fewer modes are used in $m$, mainly for the control mode. Equation (1) will be used in this case, with $m$ much smaller than $n$.

Using the above approach, nonlinear PSE computations were performed for $R_{c}=24 \times 10^{6}$, for which the linearly most unstable traveling crossflow instability mode has a frequency of approximately $1 \mathrm{kHz}$ and a spanwise wavelength of $12 \mathrm{~mm}$ (the target mode). The stationary control mode has a spanwise wavelength of $4 \mathrm{~mm}$ (one-third of the target mode). The NPSE computation is carried out in the spanwise traveling coordinate system as explained above; the target mode becomes stationary, and the control mode appears to have a frequency of $-3 \mathrm{kHz}$. It can be seen in Fig. 14 that the linear $N$ factors of the $12 \mathrm{~mm}$ target mode and the $4 \mathrm{~mm}$ control mode computed in the stationary frame and the traveling frame gave rise to the same results in each case. Thus, the switch from a stationary frame to a traveling frame does not alter the stability properties of the perturbations being analyzed.

In the control case in which both stationary and traveling modes were present, the coordinate transformation also helped save resources. Suppose the computation was carried out in the fixed frame, then the harmonics generated by the target mode alone would align themselves along the diagonal of the $(m, n)$-space, while those generated by the control mode alone would lie along the vertical axis $(0, n)$. Again, assuming that 48 modes are required to resolve the target mode, $48 \times 48$ spatial and time Fourier modes must be included in the computation. By using the moving frame of reference, the target mode harmonics are transferred to the vertical axis $(0, n)$ with $n<49$ and the control mode harmonics, being initialized at $(1,3)$ in this case, now lie along $(m, 3 m)$. As mentioned earlier, the control mode has a moderate amplitude and, therefore, just five Fourier modes are sufficient to resolve it, i.e., $m<6$, therefore, the total number of Fourier modes required is $48 \times 5$. There are, of course, nonzero off-diagonal Fourier modes in the $(m, n)$-space that are generated via interactions of the control and target modes. However, in the chordwise range in which the control mode dominates, the target mode amplitude is small, and the off-diagonal modes are of secondary importance.

Nonlinear computations for a $12 \mathrm{~mm}, 1 \mathrm{kHz}$ traveling crossflow wave are first carried out in the absence of control. The evolution of the fundamental harmonic and the mean flow correction are shown in Fig. 15(a). For comparison, the same figure also shows the results of independent calculations pertaining to the evolution of the most amplified stationary mode with a wavelength of $8 \mathrm{~mm}$. The number of Fourier modes used for the traveling mode computation is 48 , i.e., $m=0$ and $-49<n<49$ since it is done in the moving frame (for presentation purposes, the fundamental traveling mode is still referred to as mode $(1,1)$ as if it were obtained in a stationary frame). The initial amplitudes for both the stationary and traveling modes are $10^{-4}$, and these computations are done independent of each other. It should be noted that the initial amplitude of the traveling mode would realistically be much lower and it's only the higher subsequent growth that would help make the traveling mode more relevant. Consistent with linear theory, the traveling wave has a larger growth rate as an examination of the amplitude curve slopes will reveal. Interestingly, however, it is the stationary wave that reaches higher maximum amplitude than the traveling wave, approximately $50 \%$ for the former and less than $30 \%$ for the latter. The amplitudes of the mean flow correction components for both the stationary and traveling cases reach approximately $20 \%$. The faster initial growth of the 
traveling wave also drives faster growth of its harmonics, enabling nonlinear effects to set in faster, causing the traveling wave to saturate earlier, and, hence, a smaller maximum amplitude results.

Effects of control are analyzed by using 5 Fourier modes in $m$ and 48 Fourier modes in $n$. The $12 \mathrm{~mm}, 1 \mathrm{kHz}$ target mode initial amplitude is fixed at $10^{-4}$, and three different amplitudes of the $4 \mathrm{~mm}$ control mode are used, namely, 0.005, 0.01, and 0.015. Fig. 15(b) shows that, with increasing control amplitude, the rise of the target mode amplitude is progressively delayed and its peak-amplitude is also reduced, indicating that this control mechanism is very effective. This is consistent with the suggestion made in Ref. 42, based on a single low Reynolds number computation, that DRE control may also be applicable to traveling crossflow modes.

Figure 16 shows the mean velocity profiles at three selected streamwise stations in the presence of both the traveling target mode at the fixed amplitude $\left(10^{-4}\right)$ and the stationary control mode at two amplitudes $(0$ and 0.015$)$, i.e., the case studied in Fig. 15(b). The distortions to these profiles are chiefly caused by the control mode since the target mode amplitude is very small at these selected stations. The velocity profiles in the inviscid streamline direction show some velocity deficit near the boundary layer edge, which gives rise to secondary instability (Fig. 13). The crossflow profiles show reduced maximum amplitude with increasing control as noted previously for other configurations in Refs. 43 and 23, and this may be the main reason for the reduced target mode growth.

Since free-stream turbulence level in flight is low, it is reasonable to expect a much smaller initial amplitude of traveling disturbances than the surface roughness induced stationary crossflow disturbances. Therefore, another computation was performed using initial amplitude of $10^{-6}$ for the traveling mode. The results are presented in Fig. 17(a), which shows that the DRE provides an effective control of the traveling waves. The results are compared with stationary target mode with wavelength of $8 \mathrm{~mm}$ and higher initial amplitude of $10^{-4}$ in Fig. 17(b). The initial amplitude of the control mode is the same (0.015) in the two cases. Without any control, the traveling crossflow wave amplitude is already larger than that of the stationary wave at approximately $20 \%$ chord even though the former's initial amplitude is 100 times smaller than that of the latter. With control, both stationary and traveling waves are suppressed. One should note that the two results are independently generated, i.e., stationary target mode only or traveling target mode only. There would, in fact, be interactions between the stationary and traveling modes if both were present simultaneously (cf. Ref. 11). These interactions are difficult to track in the NPSE construct. Since both the stationary and traveling target modes reach large amplitudes and require a significant number of Fourier modes to resolve, no coordinate transformation will result in significant savings of computational resources.

\section{E. Secondary Instability Characteristics}

In Section C, secondary instability analysis of stationary crossflow disturbances was performed to determine effectiveness of DRE in controlling crossflow-induced transition. It was shown that the delay of transition in the presence of control mode is caused by the delay in the secondary instability growth of the target mode. The analysis also yielded interesting results about the characteristics of secondary instability modes, which are described here for both the target and control modes.

Figure 18 shows $N$ factor curves for secondary instability modes of various frequencies for the primary target and control wavelengths (obtained in independent computations). Four significant secondary instability modes are found for the target mode with initial amplitude of $10^{-4}$, while three are found for the control mode with initial amplitude of 0.015. In each case, the most amplified mode is designated as Mode 1, followed by Mode 2, etc. Secondary instability of crossflow vortices was classified in Ref. 12 into two main categories, i.e., the y-modes and z-modes. The former is associated with the strong shear layer created by the velocity-gradient in the wall-normal direction and the latter with that in the spanwise direction. For the results shown in Fig. 18, Mode 1 is a $z$-mode for the target and a $y$-mode for the control, i.e., the two types of modes correlate, respectively, with the spanwise shear and wallnormal shear induced by the primary crossflow vortex. In the low speed experiment of Ref. 44, $z$-mode of secondary instability was detected.

The various secondary instability mode growth rates for the target mode are plotted in Fig. 19 as function of frequency at a fixed streamwise station $X / C=0.3856$. This location is a short distance downstream of the station where the secondary instabilities begin to amplify and, hence, the growth rate magnitudes are still moderate at this location. The peak-growth frequency is approximately $65 \mathrm{kHz}$ for Mode 1 . Here, Mode 1 is a $z$-mode, Mode 2 a $y$ - 
mode, Mode 3 a $z$-mode, and Mode 4 a $y$-mode. Corresponding eigenfunctions near their respective peaks are plotted in Fig. 20, with the faint white lines representing the underlying stationary crossflow vortex.

Similarly, secondary instability mode growth rates for the control mode are plotted in Fig. 21 as function of frequency at $X / C=0.126$. The peak frequency is found to be approximately $90 \mathrm{kHz}$ for Mode 1 , a $y$-mode. Corresponding eigenfunctions are shown in Fig. 22.

\section{Discussion and Conclusions}

Computational analysis has been performed for a laminar flow test article designed to demonstrate discrete roughness element technology at flight Reynolds numbers of relevance to transport aircraft. The Mach number and sweep angle used in the analysis are 0.75 and $34.6^{\circ}$, respectively. Computations are performed using nonlinear parabolized stability equations and secondary instability analysis for chord Reynolds numbers of $17 \times 10^{6}, 24 \times 10^{6}$, and $30 \times 10^{6}$.

Various simplifying assumptions have been made in this study for extending swept-wing natural laminar flow using the discrete roughness elements. The infinite-swept airfoil assumption does not adequately represent the flowfield on the glove as the fully three-dimensional boundary layer on the glove is much less unstable than the infiniteswept case. In addition, the same $C_{p}$ distribution is chosen for the three flight Reynolds numbers; therefore, the results reported and conclusions drawn are specific to the selected $C_{p}$ distribution. Initial amplitudes of the target and control modes and the associated mode shapes are arbitrarily chosen. For example, linear eigen-mode was used to initialize the calculation but it is not known how far downstream of the discrete roughness element such a mode shape develops and what is the relation of its amplitude to the height and shape of the roughness. Similarly, initial target mode amplitudes are related to the specific surface finish, but these amplitudes were assumed in this study. Due to these assumptions, conclusions drawn from the present results can be characterized as qualitative at best. Definitive conclusions can only be drawn when initial modal amplitudes are available from a careful receptivity analysis for a given surface finish and DRE roughness height, shape, and location.

Computations performed in this study have demonstrated that crossflow-induced transition can be delayed if the control mode of a given wavelength and amplitude is introduced. One of the goals of the flight experiment was to demonstrate that DREs can delay transition by 50\%, which does not seem to be fully supported by these computations. Present computations show that DREs could delay transition by about $20 \%$ for the pressure distribution and flight Reynolds numbers used in the study. These results are based on secondary instability analysis of the mean flow in the presence of stationary crossflow disturbances. Several high-frequency secondary instability modes are present for the target and the control mode wavelengths. The most unstable secondary instability mode is a $z$-mode for the target, but a $y$-mode in case of the smaller wavelength control mode.

Computations were also performed to study the effect of DRE on traveling crossflow disturbances. The wavelength of the most amplified traveling disturbance (the target mode) was $12 \mathrm{~mm}$, and the stationary control mode had a wavelength of $4 \mathrm{~mm}$. The same control mode was used to delay the growth of the stationary target mode of wavelength $8 \mathrm{~mm}$. It is shown that DRE with the $4 \mathrm{~mm}$ wavelength suppresses the growth of traveling mode of frequency $1 \mathrm{kHz}$ as well.

In summary, the following conclusions can be drawn from this analysis:

- Discrete roughness elements could delay crossflow-induced transition by about $20 \%$ at Reynolds number in the range of $17 \times 10^{6}$ to $30 \times 10^{6}$.

- In addition to the stationary crossflow disturbances, discrete roughness elements are found to suppress the growth of most amplified traveling disturbances.

Acknowledgements

This work was performed in support of NASA's Environmentally Responsible Aviation (ERA) Project. 


\section{References}

1. Hefner, J. N. and Bushnell, D. M., “An Overview of Concepts for Aircraft Drag Reduction”, AGARD Report No. 654, Special Course on Concepts for Drag Reduction, pp. 1-30, 1977.

2. Marec, J-P., "Drag Reduction: a Major Task for Research," CEAS/DragNet European Drag Reduction Conference 2000, Potsdam, June 2000.

3. Washburn, A., "Drag Reduction Status and Plans-Laminar Flow and AFC," Oral Presentation at AIAA Aero Sciences Meeting, Orlando, FL, January 4-7, 2011.

4. Bushnell, D. M. and Tuttle, M. H., "Survey and bibliography on attainment of laminar flow control in air using pressure gradient and suction," Vol. I, NASA RP-1035, 1979.

5. Bushnell, D. M., "Overview of aircraft drag reduction technology," in Special course on skin friction drag reduction, AGARD Report 786, 1992.

6. Joslin, R. D., "Aircraft Laminar Flow Control," Ann. Rev. Fluid Mech., Vol. 30, 1998, pp. 1-29.

7. Green, J. E., "Laminar Flow Control-back to the Future?," AIAA Paper 2008-3738, 2008.

8. Spalart, P. R. and McLean, J. D., "Drag Reduction: enticing turbulence, and then an industry," Phil. Trans. R. Soc. A, Vol. 369, 2011, pp. 1556-1569.

9. Wilkinson, S. P. and Malik, M. R., "Stability Experiments in the Flow over a Rotating Disk," AIAA J., Vol. 23, 1985, pp. 588-595.

10. Balachandar, S., Streett, C. L., and Malik, M. R., "Secondary Instability in a Rotating Disk Flow," J. Fluid Mech., Vol. 242, 1992, pp. 323-347.

11. Malik, M. R., Li, F., and Chang, C.-L., "Crossflow disturbances in three-dimensional boundary layers: Nonlinear development, wave interaction and secondary instability," J. Fluid Mech., Vol. 268, 1994, pp. 1-36.

12. Malik, M. R., Li, F., Choudhari, M., and Chang, C.-L., "Secondary Instability of Crossflow Vortices and Swept-Wing Boundary-Layer Transition,” J. Fluid Mech., Vol. 399, 1999, pp. 85-115.

13. Kohama, Y., Saric, W. S., and Hoos, J. A., "A high-frequency secondary instability of crossflow vortices that leads to transition," in Boundary Layer Transition and Control, Proc. Royal Aeronautical Society, Cambridge, 8-12 April, 1991, London, UK.

14. White, E. B. and Saric, W. S., "Secondary instability of crossflow vortices," J. Fluid Mech., Vol. 525, 2005, pp. 275308.

15. Maddalon, D., Fisher, D., Jennett, L., and Fischer, M., "Simulated airline service experience with laminar flow control leading-edge system," NASA Symposium on NLF and LFC Research, NASA CP 24587, 1987, pp. 195-218.

16. Boeing Commercial Airplane Group, High Reynolds Number Hybrid Laminar Flow Control (HLFC) Flight Experiment Final Report. Volume I: D6-55648-1 through Volume 6: D6-55628-6, 1993.

17. Pfenninger, W., "Laminar Flow Control-Laminarization," AGARD Report No. 654, Special Course on Drag Reduction, 1977.

18. Anders, S. G. and Fischer, M. C., "F-16XL-2 Supersonic laminar Flow Control Flight Test Experiment," NASA TP1999-209683, 1999.

19. Saric, W.S., Carrillo, R. B. Jr., and Reibert, M.S., "Nonlinear stability and transition in 3-D boundary layers," Meccanica 33, 1998, pp. 469-487.

20. Chang, C.-L. and Choudhari, M., "Boundary-Layer Receptivity and Integrated Transition Prediction," AIAA Paper 2005-0526, 2005.

21. Wassermann, P. and Kloker, M., "Mechanisms and control of crossflow-vortex induced transition in a threedimensional boundary layer," J. Fluid Mech., Vol. 456, 2002, pp. 49-84.

22. Li, F., Choudhari, M. M., Chang, C.-L., Streett, C. L., and Carpenter, M. H., "Computational Modeling of RoughnessBased Laminar Flow Control on a Subsonic Swept Wing," AIAA J., Vol 49, No 3, March 2011.

23. Li, F., Choudhari, M. M., Carpenter, M. H., Malik, M. R., Chang, C.-L., and Streett, C. L., "Roughness Based Crossflow Transition Control for a Swept Airfoil Design Relevant to Subsonic Transports," AIAA Paper 2010-4380, 2010,

24. Saric, W. S., Carpenter, A. L., and Reed, H. L., "Laminar Flow Control Flight Tests for Swept Wings: Strategies for LFC," AIAA Paper 2008-3834, 2008.

25. Saric, W. S., Carpenter, A. L., and Reed, H. L., "Passive Control of Transition in Three-Dimensional Boundary Layers, with Emphasis on Discrete Roughness Elements,” Phil. Trans. R. Soc. A, Vol. 369, 2011, pp. 1352-1364.

26. Belisle, M. J., Roberts, M.W., Tufts, M. W., Tucker, A. A., Williams, T., Saric, W.S., and Reed, H. L., "Design of the Subsonic Aircraft Roughness Glove Experiment (SARGE)," AIAA Paper 2011-3524, 2011. 
27. Malik, M., Liao, W., Lee-Rausch, E., Li, F., Choudhari, M., and Chang, C.-L., "Computational Analysis of the G-III Laminar Flow Glove," AIAA Paper 2011-3525, 2011.

28. Hartshorn, F., Belisle, M. J., and Reed, H. L., "Computational Optimization of a Natural Laminar Flow Experimental Wing Glove," AIAA Paper 2012-0870, 2012.

29. Liao, W., Malik, M. R., Lee-Rausch, E. M., Li, F., Nielsen, E. J., Buning, P. G., Chang, C. L., and Choudhari, M., "Boundary-Layer Stability Analysis of the Mean Flows Obtained Using Unstructured Grids", AIAA Paper 2012-2690, 2012.

30. Roberts, M. W., Reed, H. L., and Saric, W. S., "A Transonic Laminar-Flow Wing Glove Flight Experiment: Computational Evaluation and Linear Stability,” AIAA Paper 2012-2668, 2012.

31. Nichols, R. and Buning, P., "User's Manual for OVERFLOW 2.1" NASA Langley Research Center, Hampton, VA, 2008.

32. http://fun3d.larc.nasa.gov/, Dec 4, 2012

33. Wie, Y.-S., "BLSTA - A Boundary Layer Code for Stability Analysis," NASA CR 4481, 1992.

34. Chang, C.-L., "The Langley Stability and Transition Analysis Code (LASTRAC): LST, Linear and Nonlinear PSE for 2-D, Axisymmetric, and Infinite Swept Wing Boundary Layers," AIAA Paper 2003-0974, 2003.

35. Swearingen, J. D. and Blackwelder, R. F., "The Growth and Breakdown of Streamwise Vortices in the Presence of a Wall,” J. Fluid Mech., Vol. 182, 1987, pp. 255-290.

36. Li, F. and Malik, M. R., "Fundamental and Subharmonic Secondary Instability of Görtler Vortices," J. Fluid Mech., Vol. 297, 1987, pp. 77-100.

37. Li, F. and Choudhari, M. M., "Spatially Developing Secondary Instabilities and Attachment Line Instability in Supersonic Boundary Layers," AIAA Paper 2008-590, 2008

38. Li, F. and Choudhari, M. M., "Spatially Developing Secondary Instabilities in Compressible Swept Airfoil Boundary Layers," J. Theo. Comput. Fuild Dyn. (Vol. 25, pp. 65-84, June, 2011.

39. Deyhle, H. and Bippes, H., "Disturbance Growth in an unstable three-dimensional boundary layer and its dependence on environmental conditions," J. Fluid Mech., Vol. 316, 1996, pp. 73-113.

40. Radeztsky, R. H., Reibert, M. S., Saric, W. S., and Takagi, S., "Effect of Micron-Sized Roughness on Transition in Swept-Wing Flows," AIAA Paper 93-0076, 1993.

41. Crouch, J. D. and Ng, L. L., "Variable N-Factor Method for Transition Prediction in Three-Dimensional Boundary Layers," AIAA J., Vol. 38, No. 2, 2000, pp. 211-216.

42. Choudhari, M., Chang, C.-L., Streett, C. L., and Balakumar, P., "Integrated Transition Prediction: A Case Study in Supersonic Laminar Flow Control,” AIAA Paper 2003-0973, 2003.

43. Choudhari, M., Chang, C.-L., and Jiang, L., "Towards Transition Modeling for Supersonic Laminar Flow Control," Philosophical Transactions of Royal Society of London (Physical and Mathematical Sciences), vol. 363, no. 1830, pp. 1041-1259, 2005.

44. White, E. B. and Saric, W. S., "Secondary Instability of Crossflow Vortices," J. Fluid Mech., Vol. 525, pp. 275-238, 2005. 
Table 1. Effect of DRE based control (amplitude $=0.015$ ) on delay in boundary layer transition based on final target mode amplitude of 0.25 .

\begin{tabular}{|c|c|c|c|c|}
\hline $\operatorname{Re}_{c} \times 10^{-6}$ & $A_{i n i}$ & $(X / C)_{t r}$ & $\begin{array}{c}(X / C)_{t r} \text { with } \\
\text { control }\end{array}$ & $\%$ Increment \\
\hline \multirow{3}{*}{17} & $10^{-3}$ & 0.281 & 0.306 & $9 \%$ \\
\hline & $10^{-4}$ & 0.378 & 0.434 & $15 \%$ \\
\hline & $10^{-5}$ & 0.493 & 0.591 & $20 \%$ \\
\hline \multirow{3}{*}{24} & $10^{-3}$ & 0.234 & 0.275 & $17 \%$ \\
\hline & $10^{-4}$ & 0.303 & 0.374 & $24 \%$ \\
\hline & $10^{-5}$ & 0.385 & 0.479 & $24 \%$ \\
\hline \multirow{3}{*}{30} & $10^{-3}$ & 0.157 & 0.207 & $32 \%$ \\
\hline & $10^{-4}$ & 0.212 & 0.266 & $26 \%$ \\
\hline & $10^{-5}$ & 0.271 & 0.333 & $23 \%$ \\
\hline
\end{tabular}

Table 2. DRE based control for $R e_{c}=17 \times 10^{6}$, using initial amplitude of .02 and transition location estimated using final target mode amplitude of 0.25 .

\begin{tabular}{|c|c|c|c|}
\hline $\boldsymbol{A}_{\boldsymbol{i n i}}$ & $(\boldsymbol{X} / \boldsymbol{C})_{\boldsymbol{t r}}$ & $\begin{array}{c}(\boldsymbol{X} / \boldsymbol{C})_{\boldsymbol{t r}} \text { with } \\
\text { control }\end{array}$ & \% Increment \\
\hline $\mathbf{1 0}^{\mathbf{3}}$ & 0.281 & 0.324 & $15 \%$ \\
\hline $\mathbf{1 0}^{-4}$ & 0.378 & 0.455 & $20 \%$ \\
\hline $\mathbf{1 0}^{-5}$ & 0.493 & 0.631 & $28 \%$ \\
\hline
\end{tabular}

Table 3. Effect of DRE control (amplitude $=0.015$ ) on delay in boundary layer transition based on secondary instability $N$ factor of 9 , for initial target mode amplitude of $10^{-4}$.

\begin{tabular}{|c|c|c|c|}
\hline $\boldsymbol{R e}_{\boldsymbol{c}} \times \mathbf{1 0}^{-\mathbf{6}}$ & $(\boldsymbol{X} / \boldsymbol{C})_{t r}$ & $\begin{array}{c}(X / \boldsymbol{C})_{t r} \text { with } \\
\text { control }\end{array}$ & $\%$ Increment \\
\hline $\mathbf{1 7}$ & 0.414 & 0.500 & $21 \%$ \\
\hline $\mathbf{2 4}$ & 0.329 & 0.401 & $22 \%$ \\
\hline $\mathbf{3 0}$ & 0.232 & 0.286 & $23 \%$ \\
\hline
\end{tabular}




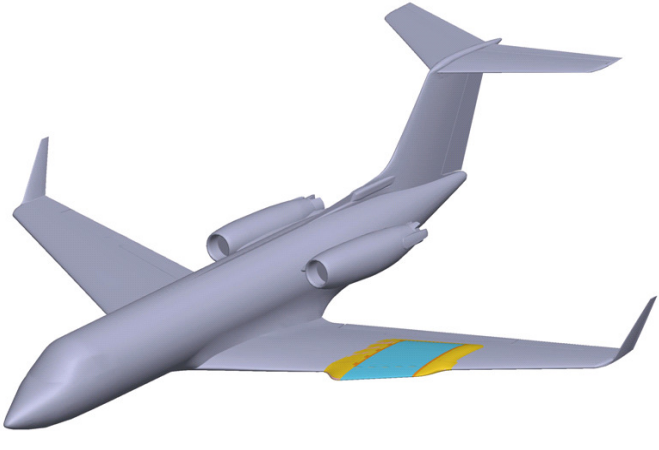

(a)

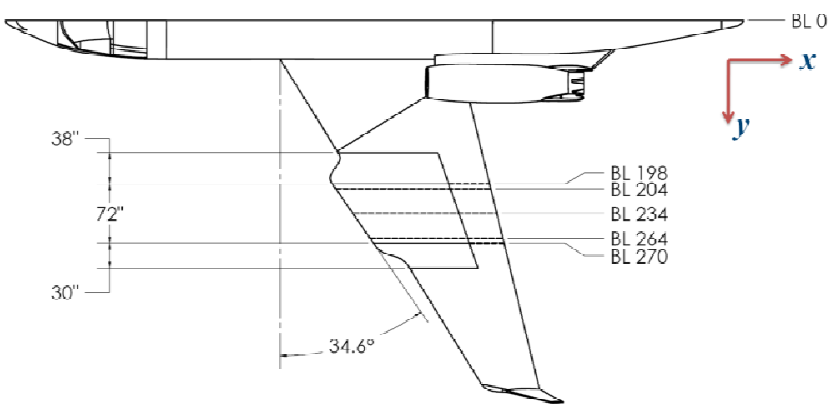

(b)

(c)

Figure 1. Gulfstream-III (G-3) aircraft and the glove. (a) Glove as installed on the G-3 wing. (b) Planform showing glove dimensions and fairings. (c) Cross section of wing-glove.

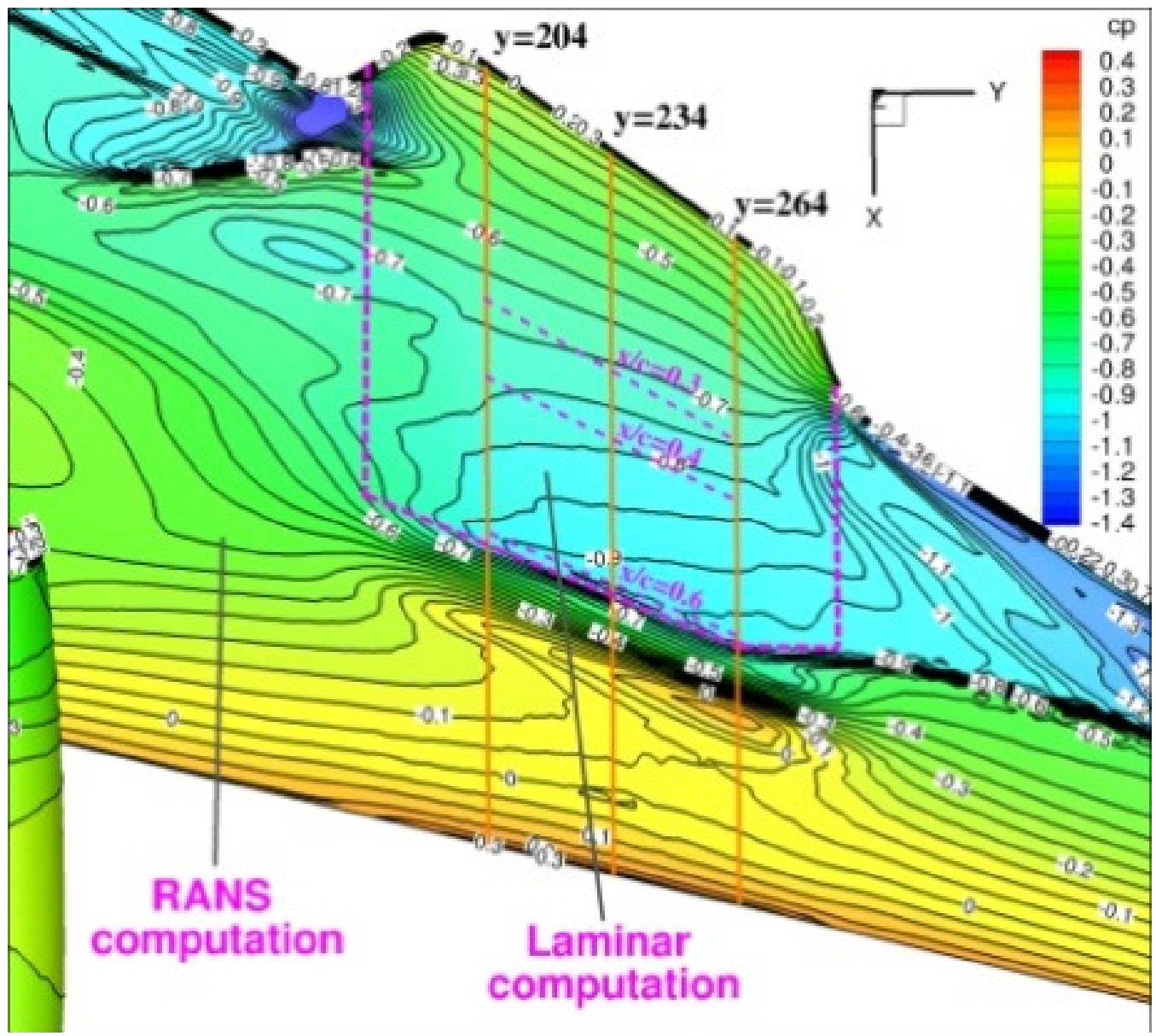

Figure 2. Upper surface $C_{p}$ distribution computed using FUN3D, assuming turbulent flow everywhere except for laminar flow on the glove up to the shock 


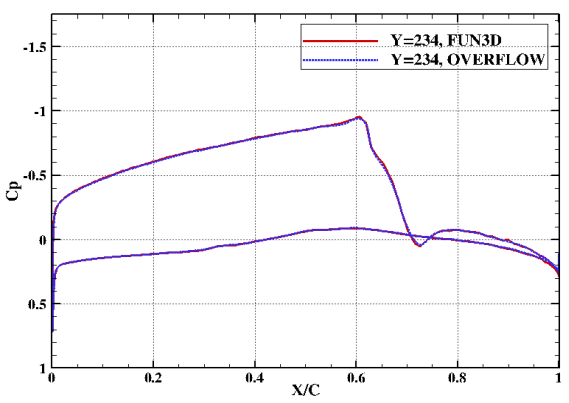

Figure 3. Computed $C p$ at the mid-span location ( $Y=234$ inches) of the glove, using FUN3D and OVERFLOW

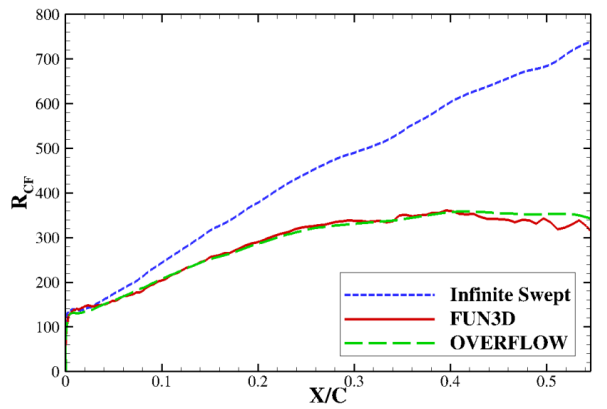

(a) Crossflow Reynolds number.

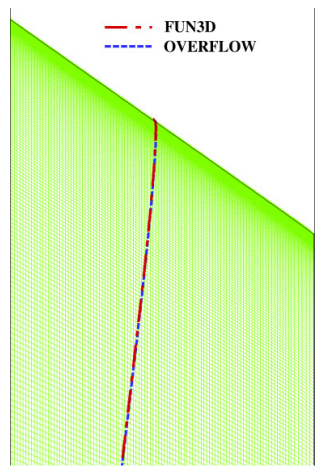

(b)Inviscid streamlines.

Figure 4. Crossflow Reynolds number.

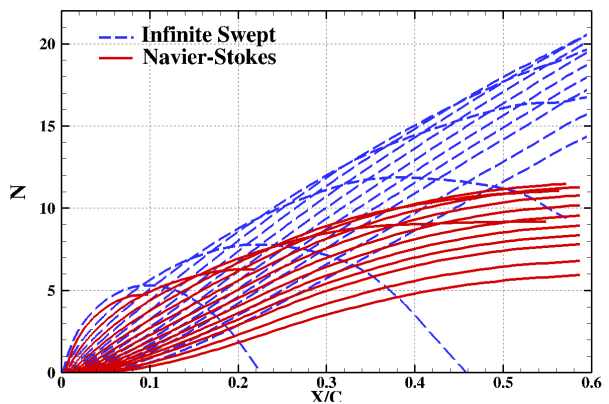

Figure 5. $N$ factors using LST with mean flow from infinite-swept boundary layer code and full FUN3D.

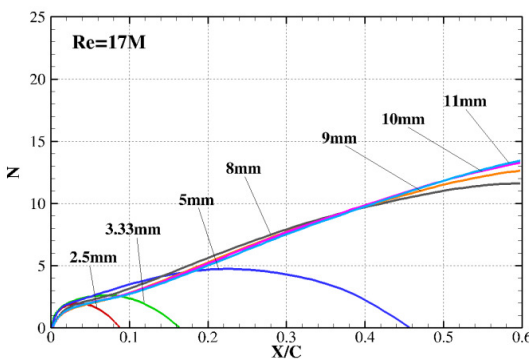

(a)

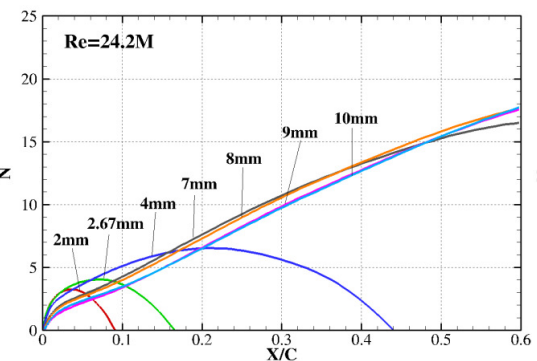

(b)

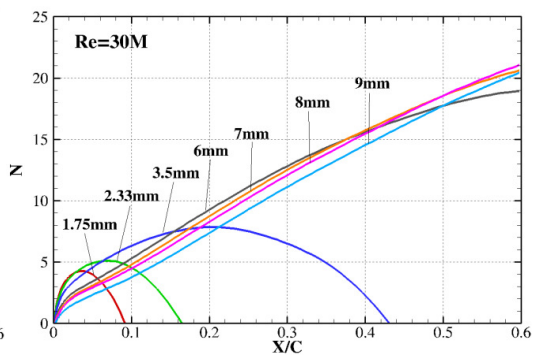

(c)

Figure 6. $N$ factors using LPSE for three different chord Reynolds numbers: (a) $17 \times 10^{6}$ (b) $24 \times 10^{6}$ (c) $30 \times 10^{6}$.

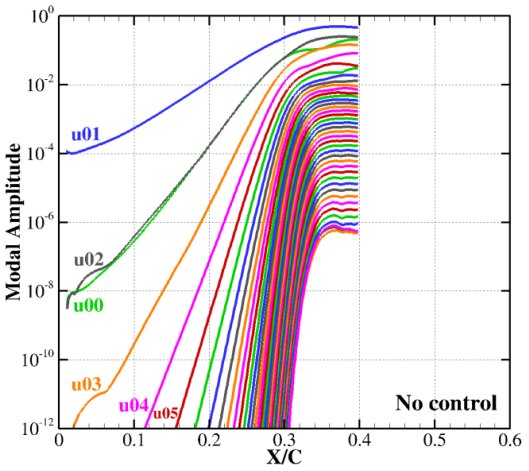

(a) No control.

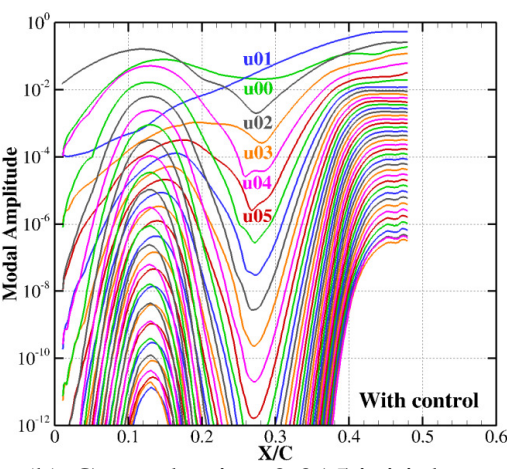

(b) Control using 0.015 initial amplitude.

Figure 7. Evolution of modal amplitudes with and without control. The target (01) mode has initial amplitude of $10^{-4}$.

The control mode is the 02 mode, with initial amplitude of 0.015 . 


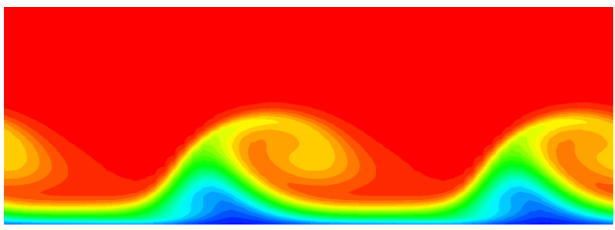

(a) No control

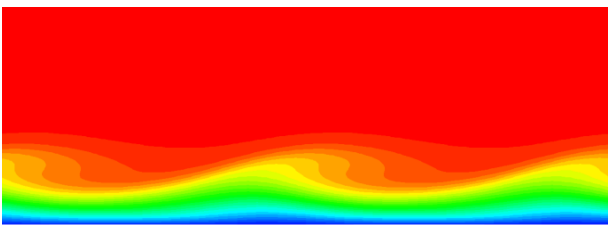

(b) Control with 0.015 initial amplitude

Figure 8. Contours of chordwise velocity over the cross section of stationary crossflow vortex at $x / c=.35$.

The horizontal (i.e., spanwise) extent of each figure corresponds to two fundamental wavelengths of the vortex.

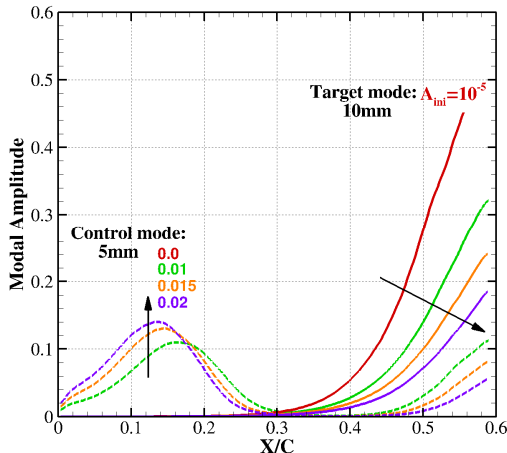

(a) $10^{-5}$

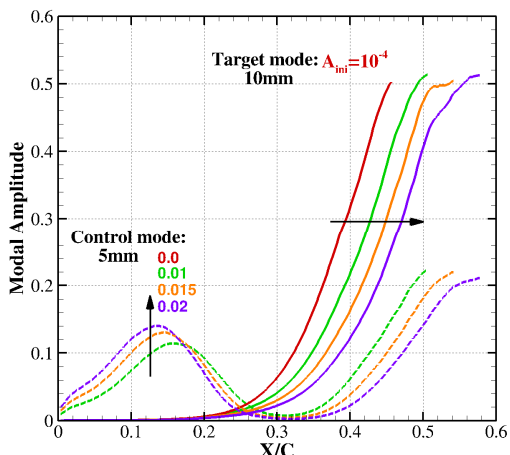

(b) $10^{-4}$

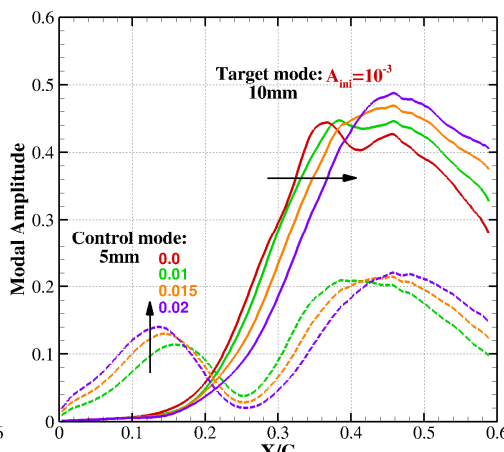

(c) $10^{-3}$

Figure 9. Nonlinear PSE results with and without control for $R e_{c}=17 \times 10^{6}$ and various initial amplitudes of the target mode.

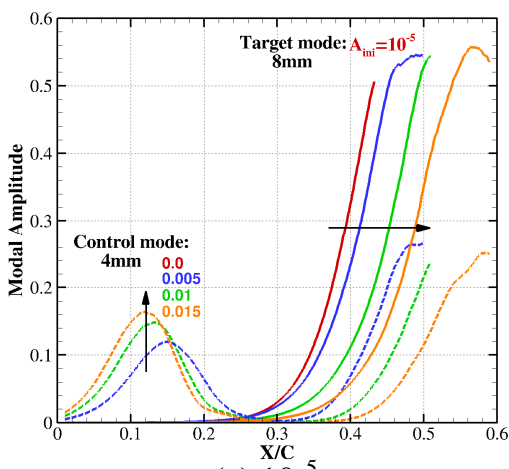

(a) $10^{-5}$

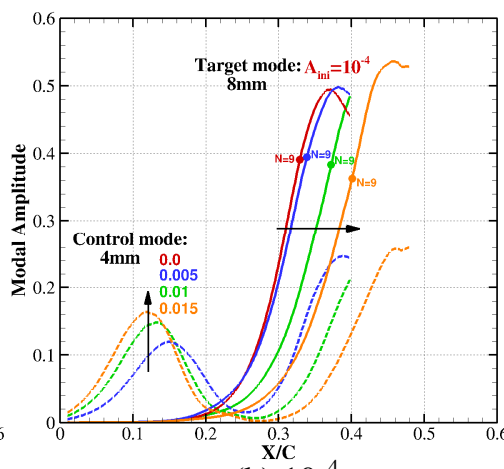

(b) $10^{-4}$

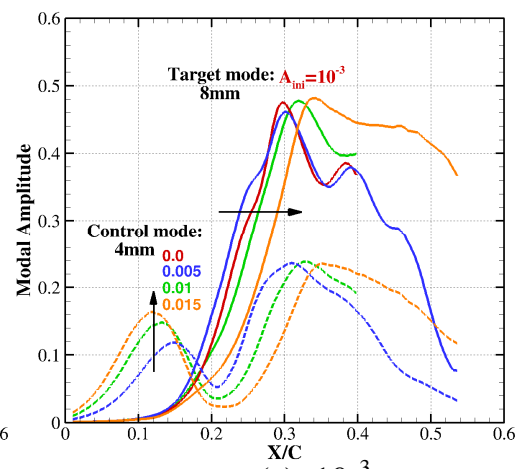

(c) $10^{-3}$

Figure 10. Nonlinear PSE results with and without control for $R e_{c}=24 \times 10^{6}$ and various initial amplitudes of the target mode.

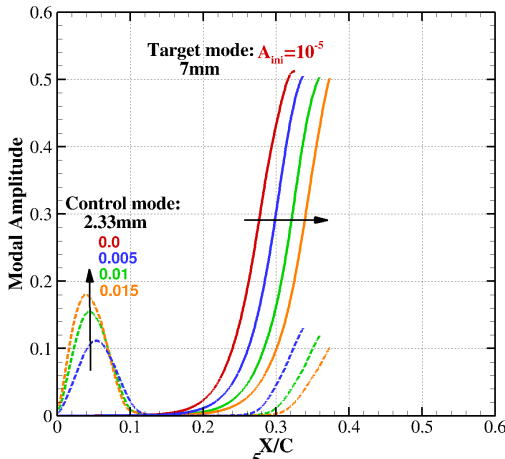

(a) $10^{-5}$

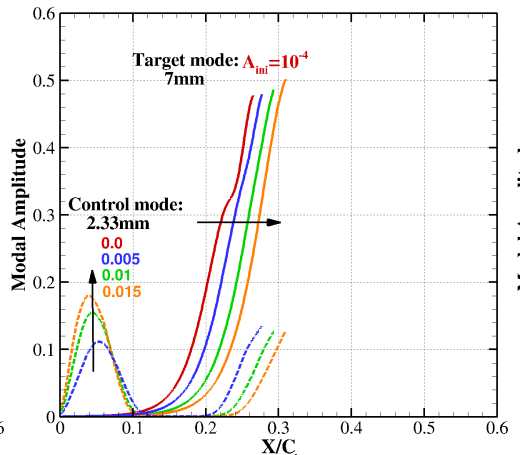

(b) $10^{-4}$

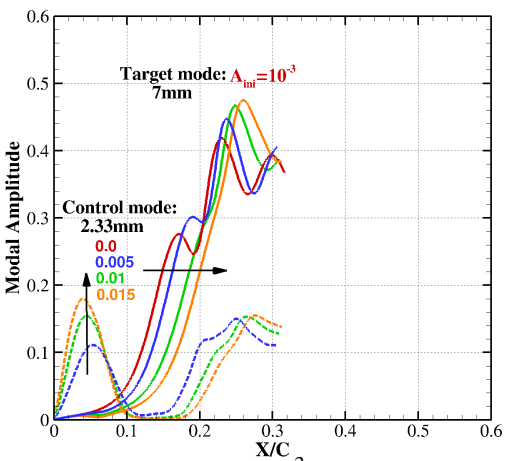

(c) $10^{-3}$

Figure 11. Nonlinear PSE results with and without control for $R e_{c}=30 \times 10^{6}$ and various initial amplitudes of the target mode. 


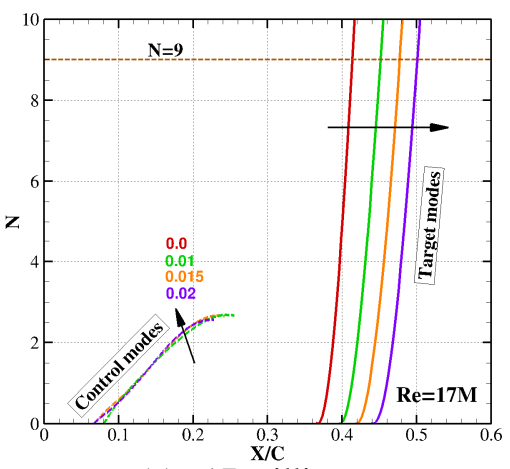

(a) 17 million.

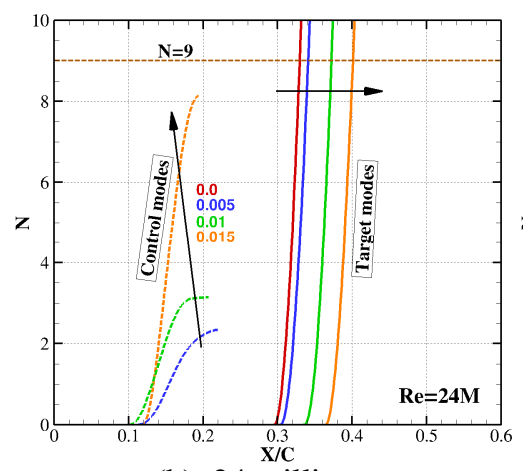

(b) 24 million.

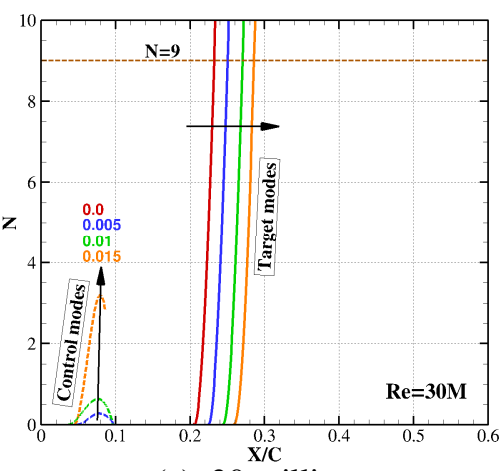

(c) 30 million.

Figure 12. $N$ factors computed using secondary instability theory for target mode amplitude of $10^{-4}$ and various initial amplitudes of the control mode.

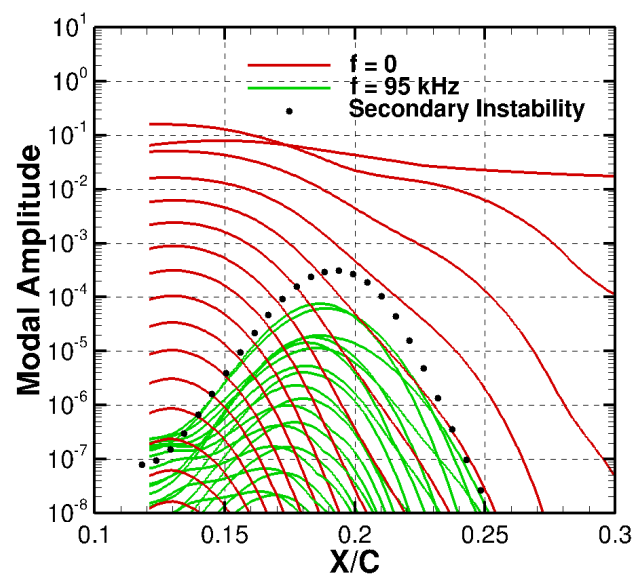

(a) Initial amplitude of secondary mode $=10^{-6}$.

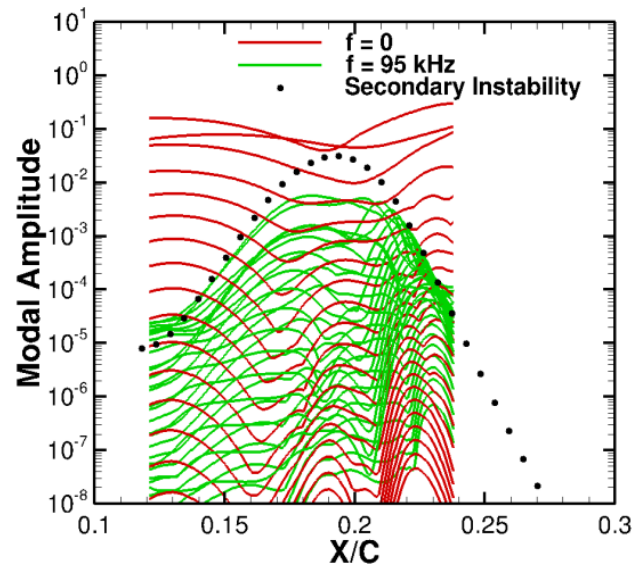

(c) Initial amplitude of secondary mode $=10^{-4}$.

Figure 13. Comparison of secondary instability results (green curve) using NPSE and linear secondary instability theory (black dot) for the control mode with amplitude of 0.015 . 


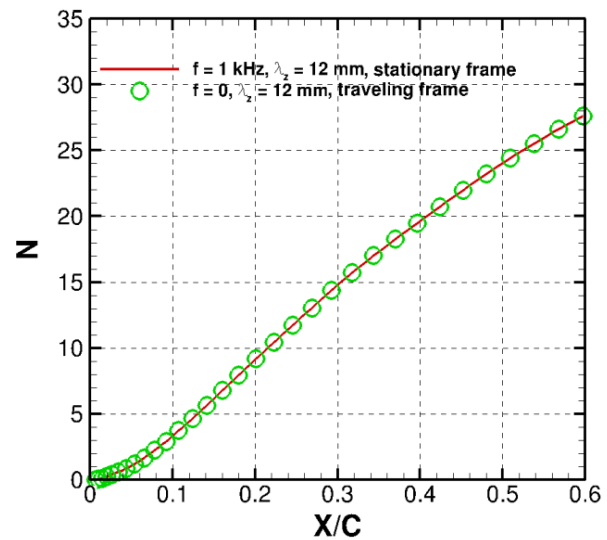

(a) Target mode

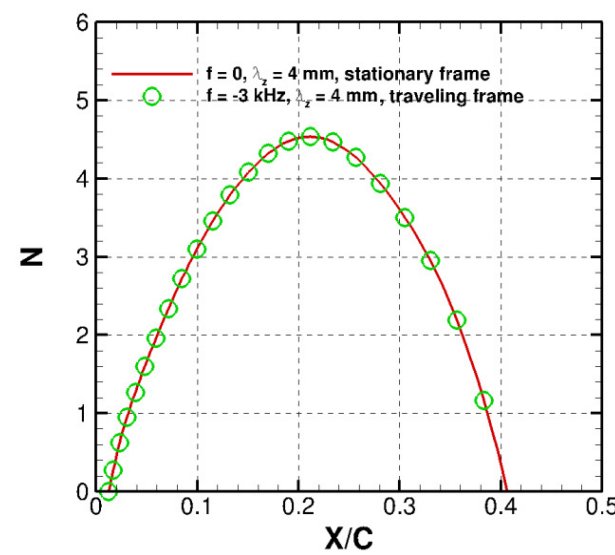

(b) Control mode.

Figure 14. Effect of coordinate transformation (Eq. 3) on traveling (target) and stationary (control) modes. Computed growth rates are independent of the transformation.

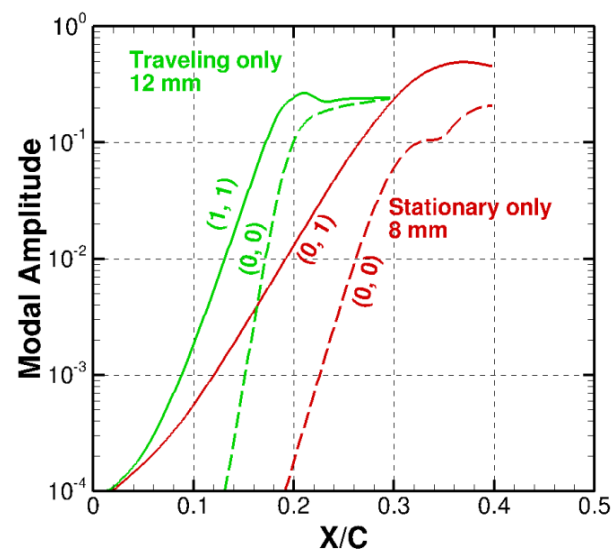

(a) Evolution of stationary and traveling crossflow waves without control. Initial amplitude: $10^{-4}$.

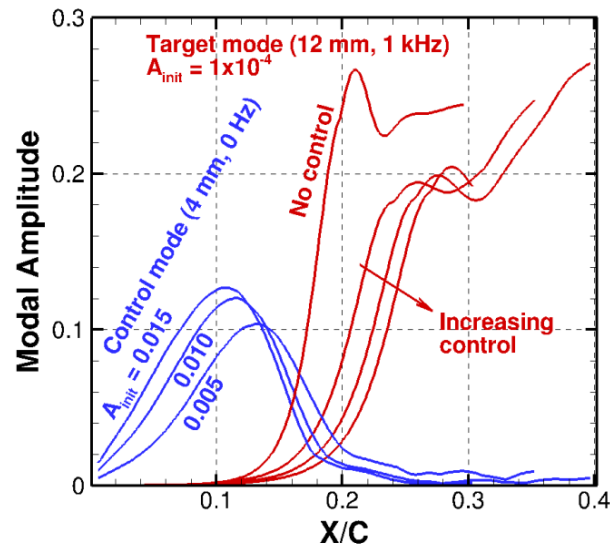

(b) Traveling crossflow wave with various controls, target mode initial amplitude: $10^{-4}$.

Figure 15. Effect of control on traveling crossflow vortices.

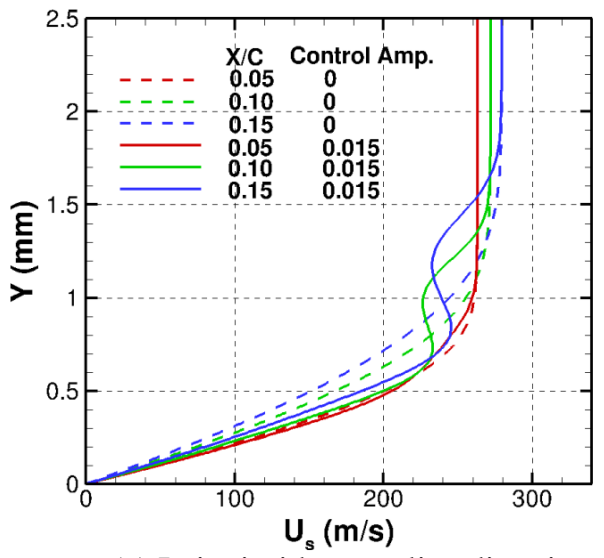

(a) In inviscid streamline direction.

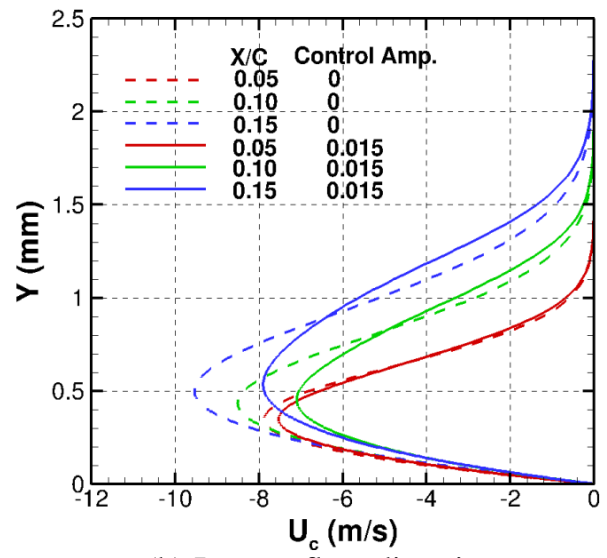

(b) In crossflow direction.

Figure 16. Mean flow velocity profiles with and without control mode at different stations. Traveling crossflow initial amplitude is $10^{-4}$. 


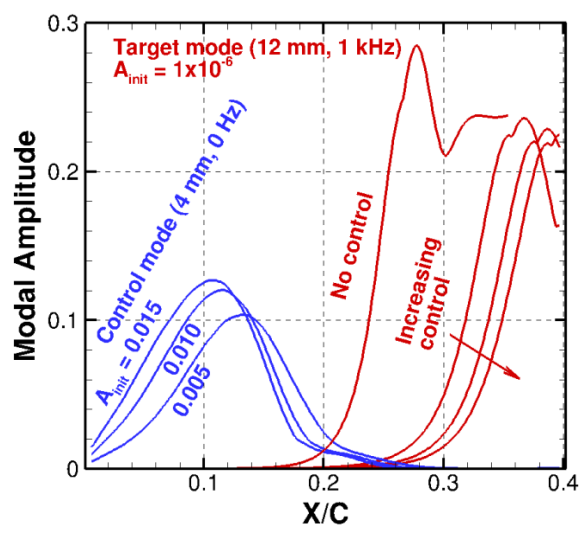

(a) Traveling crossflow wave with various control; target mode initial amplitude: $10^{-6}$.

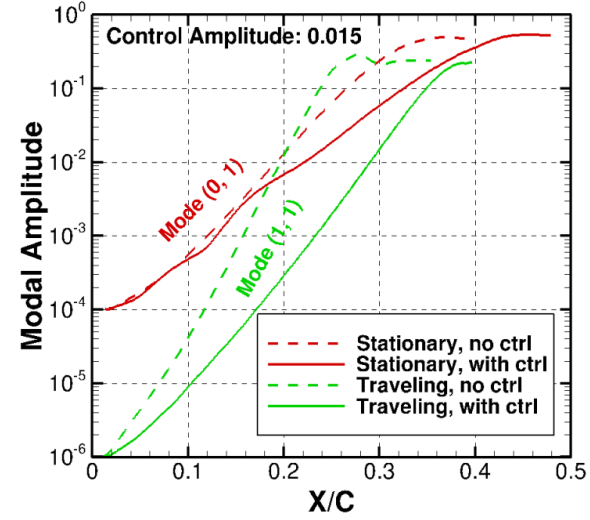

(b) Comparison of nonlinear evolution of stationary and traveling modes with and without control; initial amplitude of stationary mode $=10^{-4}$, amplitude of traveling mode $=10^{-6}$.

Figure 17. Effect of control on traveling crossflow vortices.

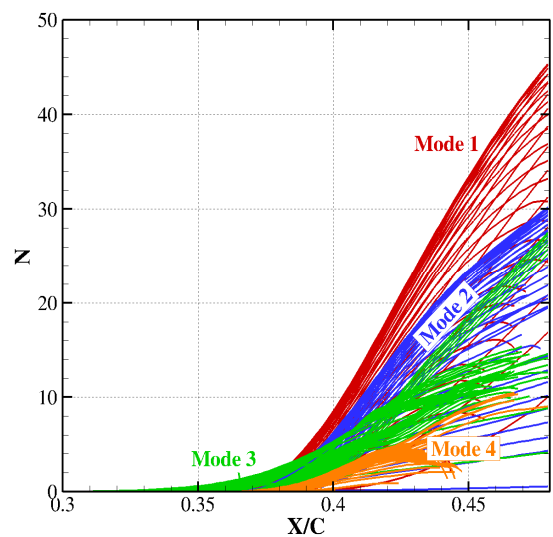

(a) $8 \mathrm{~mm}$ target mode, no control, initial amplitude $10^{-4}$.

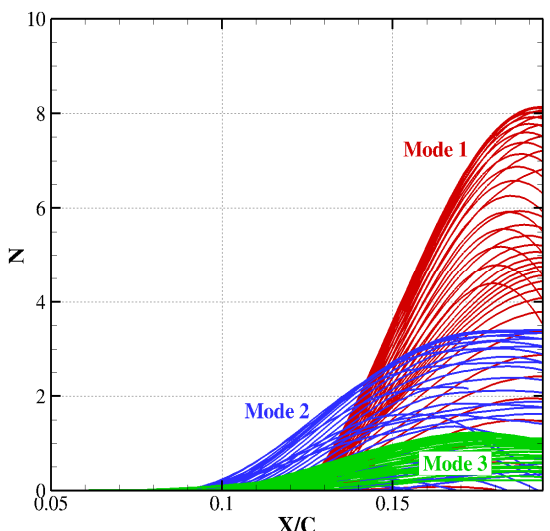

(b) $4 \mathrm{~mm}$ control mode, no target, initial amplitude 0.015 .

Figure 18. Secondary instability $N$ factors for target and control modes. Each curve is associated with a fixed frequency. Different colors represent different secondary instability modes.

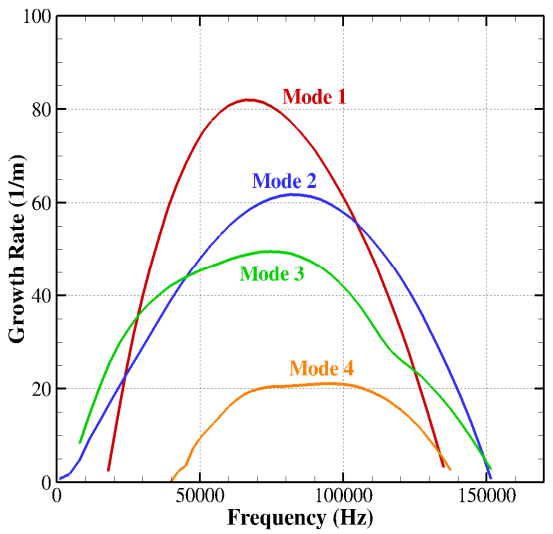

Figure 19. Growth rate spectra of secondary instability for target mode of initial amplitude $10^{-4}$ and without control at $X / C=0.3856$. 


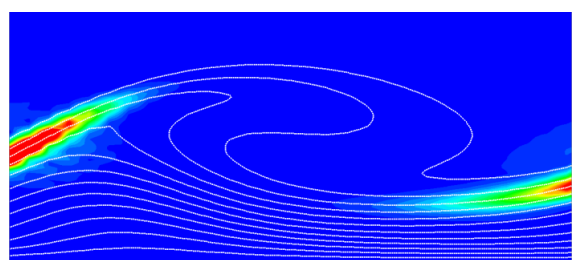

(a) Mode 1: a z-mode.

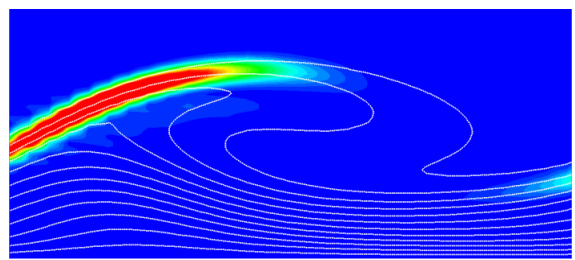

(c) Mode 3: a Z-mode.

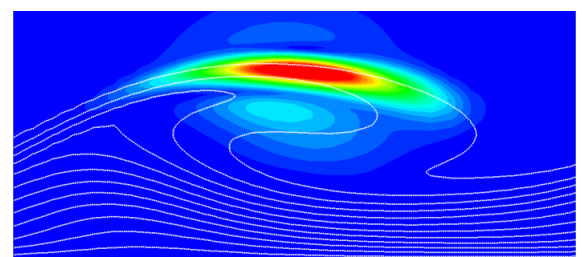

(b) Mode 2: a $y$-mode.

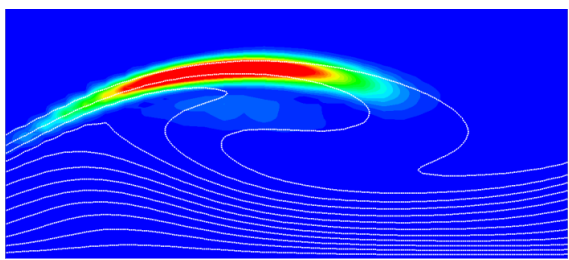

(d) Mode 4: a $Y$-mode.

Figure 20. Normal chordwise-velocity eigenfunctions for the four modes of Fig.19. Faint white lines in the background indicate the crossflow vortex.

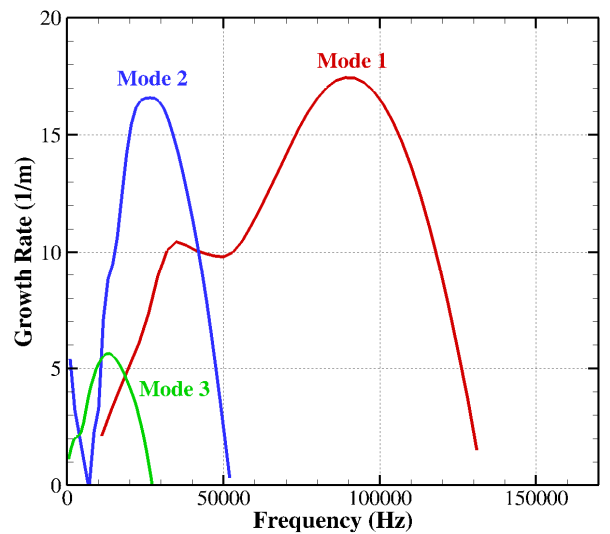

Figure 21. Growth rate spectra of secondary instability for control mode with initial amplitude 0.015 at $X / C=0.126$

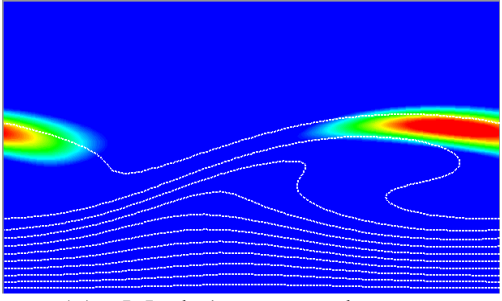

(a) Mode1: a $y$-mode.

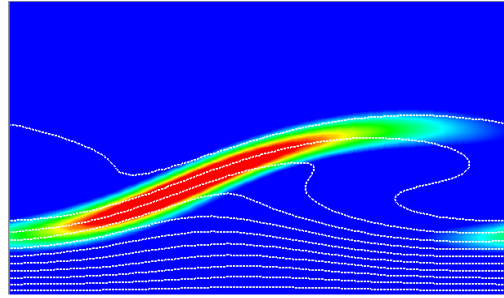

(b) Mode 2: a $z$-mode.

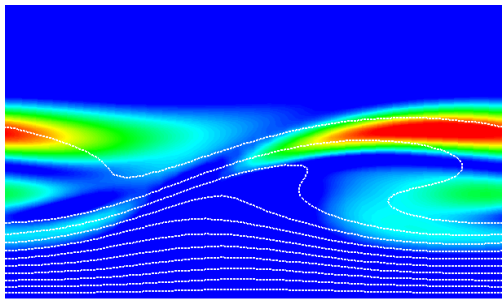

(c) Mode 3: a $Y$-mode.

Figure 22. Normal-chordwise velocity eigenfunctions for the three modes of Fig. 21. 